\title{
BMJ Open Auricular points acupressure for insulin resistance in overweight/obese women with polycystic ovary syndrome: protocol for a randomised controlled pilot trial
}

\begin{tabular}{l}
\hline Yan Li, ${ }^{1}$ Lihui Hou, ${ }^{1}$ Yingji Wang, ${ }^{2}$ Liangzhen Xie, ${ }^{\odot 1}$ Meiwei Zhang, ${ }^{3}$ Zimeng Pan, ${ }^{3}$ \\
Yangyang Li, ${ }^{3}$ Yicheng Ding, ${ }^{3}$ Miao Sun, ${ }^{1}$ Yufang Qu, ${ }^{4}$ Songjiang Liu ${ }^{\oplus}$
\end{tabular}

To cite: Li Y, Hou L, Wang Y, et al. Auricular points acupressure for insulin resistance in overweight/ obese women with polycystic ovary syndrome: protocol for a randomised controlled pilot trial. BMJ Open 2019;9:e27498. doi:10.1136/ bmjopen-2018-027498

- Prepublication history and additional material for this paper are available online. To view these files, please visit the journal online (http://dx.doi. org/10.1136/bmjopen-2018027498).

Received 25 October 2018 Revised 22 March 2019 Accepted 26 March 2019

Check for updates

(c) Author(s) (or their employer(s)) 2019. Re-use permitted under CC BY-NC. No commercial re-use. See rights and permissions. Published by BMJ.

${ }^{1}$ First Affiliated Hospital, Heilongjiang University of Chinese Medicine, Harbin, China ${ }^{2} \mathrm{School}$ of Pharmacy, Harbin Medical University, Harbin, China ${ }^{3}$ Heilongjiang University of Chinese Medicine, Harbin, China ${ }^{4}$ Qiqihar Traditional Chinese Medicine Hospital, Qiqihar, China

Correspondence to

Dr Songjiang Liu;

liusongjiang667@126.com

\section{ABSTRACT}

Introduction Approximately 5\%-20\% of reproductive women suffer from polycystic ovary syndrome (PCOS). Auricular points acupressure $(A A)$ may serve as alternative management for PCOS for its benefits in both physical and psychological well-being. However, the effects of AA for insulin resistance $(\mathrm{IR})$ in overweight/obese PCOS women have not been confirmed.

Methods and analysis The present study is designed as a randomised, placebo-controlled pilot trial to evaluate the effectiveness and safety of $A A$ in treating IR in women with PCOS. A total of 60 eligible PCOS subjects will be randomised into an intervention group (AA group) and a control group (sham AA group) in a ratio of 1:1. Magnetic beads will be taped to the auricular points by the same senior acupuncture specialist from the First Affiliated Hospital, Heilongjiang University of Chinese Medicine. The treatment will last for 12 weeks. Primary outcome measure will be changes in homeostasis model assessment of IR between baseline and after 3 months of $A A /$ sham $A A$ treatment. Secondary outcomes include hormonal profile, weight, waist/hip circumference, body mass index, blood pressure, Ferriman-Gallwey score, acne and the assessment of health-related quality of life. Outcome measures are collected at baseline and the end of treatment visit.

Ethics and dissemination The protocol has been approved by the ethics committee of the First Affiliated Hospital of Heilongjiang University of Chinese Medicine (HZYLLKY201800301). Written informed consent will be obtained from all participants. The results will be disseminated through peer-reviewed journals for publications.

Trial registration number NCT03546595; Pre-results.

\section{INTRODUCTION}

Polycystic ovary syndrome (PCOS) is a common endocrine disorder impacting $5 \%-20 \%$ reproductive women, its main symptoms include infertility, menstrual dysfunction, hirsutism and obesity, which not only
Strengths and limitations of this study

- A randomised, sham-controlled pilot trial.

- The first to investigate the effectiveness of auricular points acupressure for insulin resistance in overweight/obese women with polycystic ovary syndrome (PCOS).

- Both physical and psychological measurements of PCOS were employed.

- The results may have been underpowered due to small sample size.

cause physical dysfunction but also psychosocial stress. ${ }^{1}$

Insulin resistance (IR) and compensatory hyperinsulinemia play an important role in the pathogenesis of PCOS; hence, insulin-sensitising agents including metformin and thiazolidinediones are wildly used in treating PCOS. ${ }^{2}$ In the past two decades, metformin has gained a great deal of interest from researchers because it has the capability to ameliorate IR and it has potential reproductive benefits in women with PCOS. A review summarised current research on the effects of metformin in women with PCOS showed that metformin has beneficial effects as a cotreatment in increasing clinical pregnancy rates and reducing the risk of ovarian hyperstimulation syndrome in PCOS patients undergoing assisted reproduction techniques. ${ }^{3}$ However, the available evidence is insufficient to recommend metformin as a primary treatment in non-obese women with PCOS. ${ }^{3}$ Moreover, the high incidence of gastrointestinal symptoms leads to poor compliance in its clinical use. Thiazolidinediones including troglitazone, rosiglitazone and pioglitazone are not commonly used due to their potential 


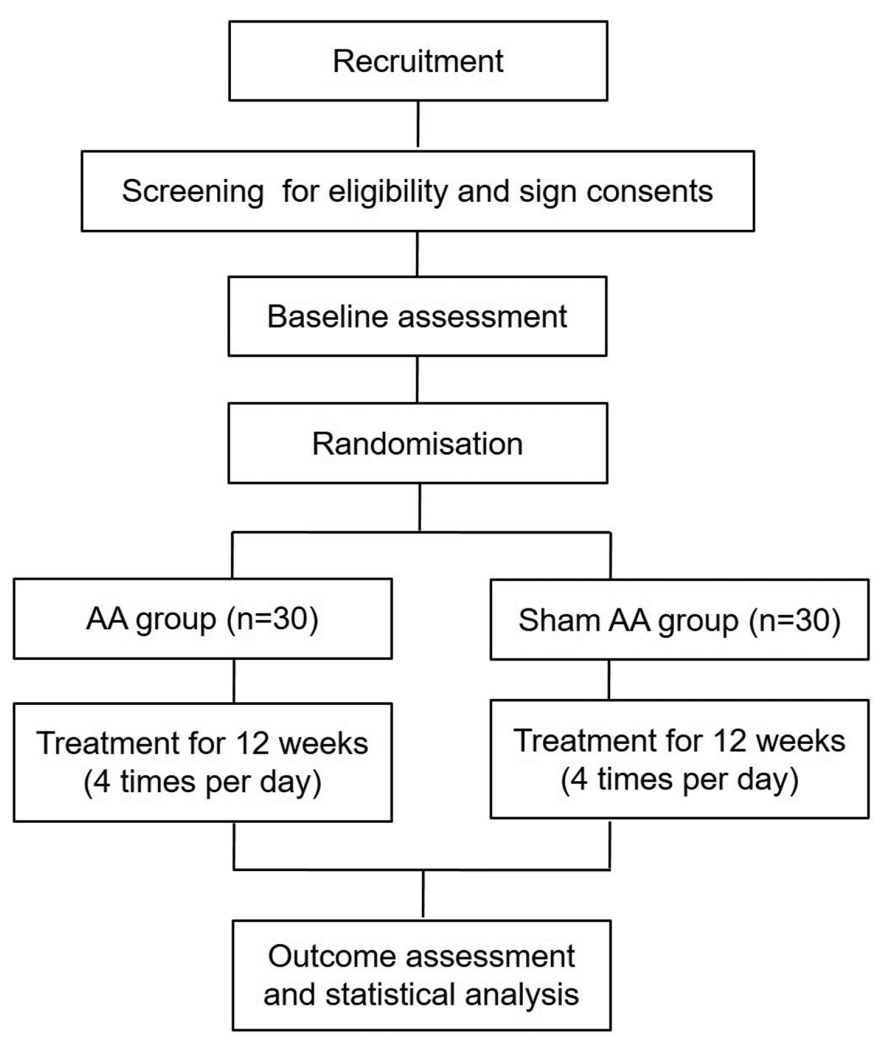

Figure 1 Study design flowchart. AA, auricular points acupressure.

risk of hepatotoxicity, causing fetal growth restriction and weight gain. ${ }^{4-7}$

Auricular points acupressure (AA) is a therapeutic approach that treats various disorders by stimulating specific points on the ear; it is a popular form of complementary medicine that has long been used in China. Since French practitioner, Paul Nogier discovered the inverted-fetus shape distribution pattern of auricular acupoints, AA has made rapid progress in the medical practice.

AA applies vaccaria seeds or steel beads on auricular acupoints to stimulate cranial nerves/spinal nerve on the auricle of the ear which causes a reaction in the central nervous system. Although there is no evidence concerning AA on insulin sensitivity in PCOS women, there are studies reporting the effect of acupuncture. A randomised controlled trial (RCT) compared abdominal acupuncture with metformin in 86 obesity PCOS subjects and found that both abdominal acupuncture and metformin could significantly decrease homeostatic model assessment (HOMA-IR) and there was no significant difference between groups. ${ }^{8}$ Chen et a ${ }^{\theta}$ reported an RCT of embedding therapy on backshu points and frontmu points combined with needle pricking therapy on Sifeng (EX-UE 10) compared with metformin in 85 PCOS subjects. Also, Yang et $a l^{10}$ reported acupuncture + Chinese medicine + letrozole + metformin compared with letrozole + metformin administration in 60 PCOS subjects, both studies showed that HOMA-IR was decreased significantly in the acupuncture group.
A recent published meta-analysis reported that the pooled results showed that acupuncture had significant effects on HOMA-IR (mean difference [MD] 0.70, $95 \%$ CI 0.04 to $1.35, \mathrm{p}=0.04<0.05$ ) in 9 RCTs involving 562 subjects suffering from multiple diseases; however, the 3 above-mentioned studies showed that reduction in HOMA-IR in PCOS was not statistically significant (MD $-0.05,95 \% \mathrm{CI}-0.17$ to $0.07, \mathrm{p}=0.44>0.05$ ). Well-designed RCTs are needed to further clarify the effect of acupuncture on IR in PCOS women and there are some ongoing trials registered. ${ }^{11}$ A prospective pilot trial (NCT02026323) designed to evaluate acupuncture on insulin sensitivity in 81 cases of women with PCOS with IR showed that acupuncture has a significant effect on improving the insulin sensitivity in these patients, and an ongoing RCT is conducted by the same research group to confirm the effects of acupuncture on the insulin sensitivity of women with PCOS combined with IR (NCT02491333). ${ }^{12}$ Stener-Victorin et al reported a protocol (NCT02647827) to investigate if acupuncture treatment or metformin together with lifestyle or lifestyle management alone improves insulin sensitivity and related symptoms in overweight/obese women with PCOS. Hopefully, these ongoing trials will provide strong evidence to support using acupuncture in treating PCOS. ${ }^{13}$

A clinical trial in persons with high-risk diabetes mellitus showed that AA could increase the concentration of antioxidative enzymes and alleviate oxidative stress, which is also very important in the pathology of PCOS. ${ }^{14}$ The mechanisms of AA in treating metabolic disorders are still not clear. Studies report that stimulation of the auricular regions is associated with the ventromedial hypothalamus, which affects the satiety centre and leads to weight loss. ${ }^{15}$ Stimulation on auricular acupoints may increase the secretion of ghrelin, which is a peptide hormone that induces satiation and is inversely related to caloric intake; it could also decrease leptin levels which is positively associated with body mass index (BMI) and IR in PCOS patients. ${ }^{16} 17$

PCOS-related symptoms and complications can lead to mood disorders, eating disorders, social and marital conflicts, and sexual dysfunction, ${ }^{18-21}$ and they were confirmed to lead to significant reduction in quality of life (QoL).$^{22}{ }^{23}$ Recent studies demonstrate the favourable effect of AA for improvement of QoL in patients with ischaemic heart diseases, breast cancer, constipation and diabetic patients with chronic kidney diseases, ${ }^{24-27}$ but whether AA is also effective in improving QoL in women with PCOS is still unknown.

AA is non-invasive and easy to apply. It can be self-administered and suitable for chronic diseases such as PCOS. To the best of our knowledge, there is no study that has investigated the effect of AA in women with PCOS. Therefore, the purpose of this pilot study is to investigate the clinical effectiveness of AA on IR in women with PCOS. The result of the present study will provide evidence to perform a larger multicentre RCT in the future. 
Table 1 Schedule of enrolment, interventions and assessments

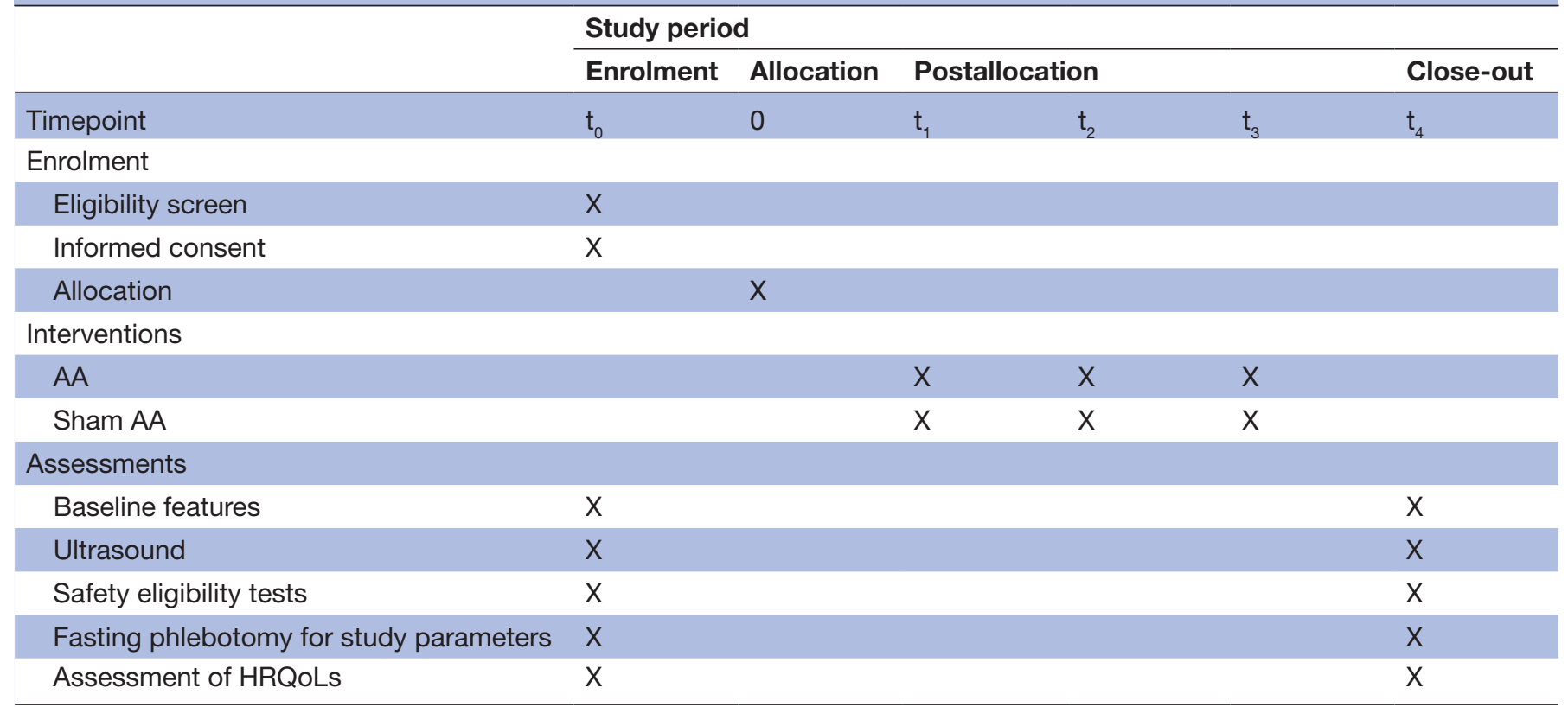

Baseline features include HOMA-IR (primary outcome), hormonal profiles (T, AND, SHBG, DHEAS, FSH, LH, $E_{2}$ ), weight, waist/hip circumference, BMI, blood pressure, FG score and acne. Safety eligibility includes liver function, renal function and complete blood count. t0, enrolment; t1, 1 month after allocation; t2, 2 months after allocation; t3, 3 months after allocation, t4, the beginning of the fourth month after allocation.

AA, auricular points acupressure; AND, androstadienedione; BMI, body mass index; DHEAS, dehydroepiandrosterone sulfate; $E_{2}$, estradiol; FG, Ferriman-Gallwey; FSH, follicle-stimulating hormone; HOMA-IR, homeostatic model assessment ; HRQoL, health-related quality of life; $\mathrm{LH}$, luteinising hormone; SHBG, sex hormone-binding globulin; T, testosterone.

\section{METHODS AND ANALYSIS Study design}

This is a parallel randomised pilot trial designed to evaluate the effectiveness of AA in treating IR for women with PCOS and safety during treatment. Sixty participants diagnosed with PCOS will be recruited from First Affiliated Hospital, Heilongjiang University of Chinese Medicine. The study period is 3 months. A flowchart of the study is depicted in figure 1 . The schedule of trial enrolment, interventions and assessments is provided in table 1. This protocol was written following the Standard Protocol Items: Recommendations for Interventional Trials checklist (see online additional file 1) and Standards for Reporting Interventions in Clinical Trials of Acupuncture: extending the Consolidated Standards of Reporting Trials statement.

\section{Inclusion criteria}

1. Women aged between 15 and 40 years.

2. Diagnosis of PCOS according to the modified Rotterdam criteria.

3. 2 years after menarche.

4. $\mathrm{BMI} \geq 23 \mathrm{~kg} / \mathrm{m}^{2}{ }^{28}$

5. IR is defined by the homeostatic model assessment (HOMA-IR: (fasting insulin $(\mu \mathrm{U} / \mathrm{mL}) \times$ fasting glucose $(\mathrm{mmol} / \mathrm{L})) / 22.5)$. A value $\geq 2.14$ will be considered to be indicative of IR. ${ }^{29}$

6. With no desire for children within 3 months.
PCOS is defined by the modified Rotterdam criteria: oligomenorrhea or amenorrhea, together with the presence of $\geq 12$ antral follicles $(\leq 9 \mathrm{~mm})$ and/or ovarian volume $>10 \mathrm{~mL}$ on transvaginal scanning, and/or clinical/biochemical hyperandrogenism. Oligomenorrhea is defined as an intermenstrual interval $>35$ days and $<8$ menstrual bleedings in the past year. Amenorrhea is defined as an intermenstrual interval $>90$ days. Clinical hyperandrogenism in China Mainland is defined as a Ferriman-Gallwey (FG) score $\geq 5 .^{30}$

\section{Exclusion criteria}

1. Administration of other medications known to affect reproductive function or metabolism within the past 3 months, including oral contraceptives, gonadotropin-releasing hormone agonists and antagonists, antiandrogens, gonadotropins, antiobesity drugs, Chinese herbal medicines, antidiabetic drugs such as metformin and thiazolidinediones, somatostatin, diazoxide and calcium channel blockers.

2. Patients with other endocrine disorders including 21-hydroxylase deficiency, hyperprolactinemia, uncorrected thyroid disease, suspected Cushing's syndrome.

3. Patients with known severe organ dysfunction or mental illness.

\section{Interventions}

Eligible patients will be randomised into one of the two arms: AA group and sham AA group. In both groups, 


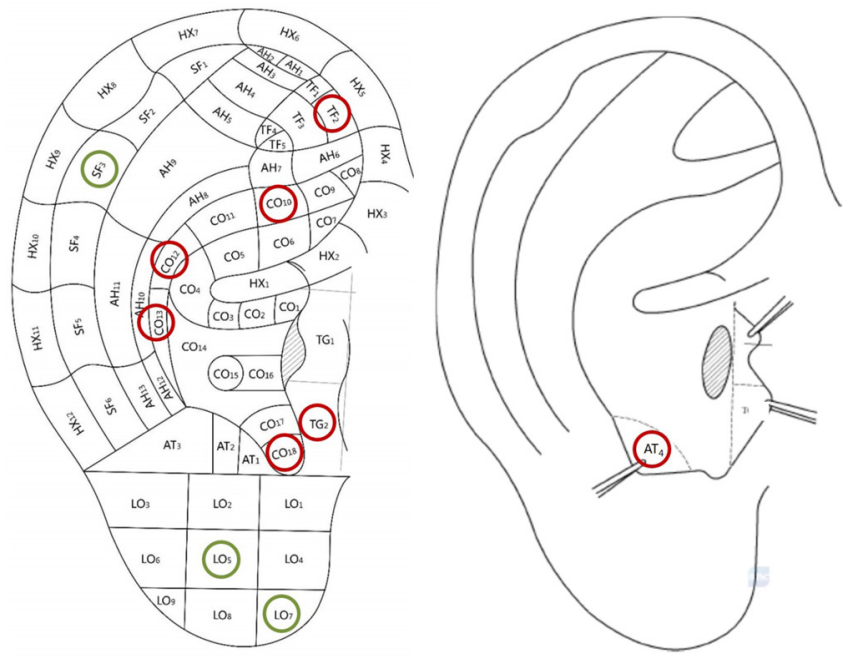

Figure 2 Locations of auricular acupoints applied in AA group (red circles) and sham AA group (green circles). AA, auricular points acupressure.

magnetic beads (Hengshui Fengfan Medical Devices Co.) were taped to the auricular acupressure points, by the same senior acupuncture specialist who has a license to practice acupuncture and has been trained to practice AA.

Based on literature and expert experience, participants in the treatment group (AA group) will receive AA on the following seven acupoints: CO10 (Shen), CO12 (Gan), CO13 (Pi), CO18 (Nei fen mi), AT4 (Pi zhixia), TF2 (Nei sheng zhi qi) and TG2 (Xia ping) ${ }^{15-17}$; participants in the control group (sham AA group) will receive AA on the following three acupoints: LO5 (Yan), LO7 (Bian tao ti) and SF3 (Zhou) (figure 2). Sham acupoints were chosen because they will have no therapeutic effects in treating PCOS or PCOS-related conditions. ${ }^{31}$ The auricular points' names and locations follow the 'Nomenclature and location of auricular points' (GB/T 13734-2008, http://www.gb688.cn/bzgk/gb/newGbInfo?hcno=D6A1 25ED4011A9A625F8A3ACE425EE09) published by the Standardization Administration of People's Republic of China.

The subjects were asked to massage the auricular points with their hands four times per day, which are $30 \mathrm{~min}$ before three meals and sleep. Each time lasts at least $5 \mathrm{~min}$; the subjects should feel sourness and heat of the ear as the response sought. The magnetic beads will be left in situ but replaced by the same acupuncture specialist with new ones once every week in the other ear. Adherence to massage over the magnetic beads was tracked via self-report logs, which was obtained at the weekly frequency during AA/sham AA treatment.

\section{Patient and public involvement}

The present trial was developed by investigators and acupuncturists based on previous clinical experience and literature. Patients were not involved in the design of the study. The outcomes were commonly used assessments of PCOS in clinical practice. The cost of interventions and outcome measurements were mostly covered by the study funding so it was not thought to be a significant burden and were in line with patient preferences. Study participants will receive a summary of her own study data and the study results once they have been published.

\section{Outcome measures \\ Primary outcome}

The primary outcome will be the changes in HOMA-IR between baseline and after 3 months of AA/sham AA treatment. Fasting glucose and insulin level will be determined.

\section{Secondary outcome}

- Hormonal profile including testosterone, androstadienedione, sex hormone-binding globulin, dehydroepiandrosterone sulfate, follicle-stimulating hormone, luteinising hormone and estradiol.

- Weight, waist/hip circumference, BMI, blood pressure, FG score and acne lesion counts.

- The assessment of health-related quality of life including short-form health survey, the Chinese Quality of Life questionnaire and health-related quality of life questionnaire for polycystic ovary syndrome. ${ }^{32-34}$

- Adverse events. Adverse events will be collected during the weekly treatment visits or through phone calls.

All the outcome assessment will be done at baseline visit and repeated at end-of-treatment visit except for the adverse events.

\section{Sample size calculation}

Most methodological papers that focus on recommendations about sample size requirements for pilot trials assume that the main aim of such a trial is to estimate a quantitative measure such as the variance (or SD) of an effect size to inform the sample size calculation for a future definitive RCT. Methods focus on the precision with which such estimates can be obtained. Whitehead $e t$ $a l$ suggest that the size of a pilot trial should be related to the size of the future definitive RCT. For such a trial designed with $90 \%$ power and two-sided $5 \%$ significance, they recommend pilot trial sample sizes for each treatment arm of 75, 25, 15 and 10 for standardised effect sizes that are extra small $(0.1)$, small $(0.2)$, medium $(0.5)$ or large $(0.8)$, respectively. ${ }^{35}$ The present study is designed with $90 \%$ power and two-sided 5\% significance, and sample size for each treatment arm is set to 25 subjects with the allocated ratio of 1:1. With an estimated 20\% drop out rate, the recruitment plan was 30 subjects per group.

\section{Randomisation and allocation concealment}

After the baseline evaluation, 60 subjects were allocated randomly into one of the two groups in a ratio of 1:1. The identification code and random number, which were unique for each subject, were generated using SAS V.9.4 by an independent agency (TCM Translational Medicine Research Centre, First Affiliated Hospital, Heilongjiang 
University of Chinese Medicine). Personnel involved in the sequence generation or allocation concealment steps were not involved in the implementation step. These assignments were put into sequentially numbered, sealed, opaque envelopes. After the investigator had obtained the patient's consent, he/she would contact the above-mentioned agency, and the agency would hand out the envelope sequentially to the investigator. Subject's name and other details were written on the envelope by the investigator and the enveloped kept sealed until it was passed onto the acupuncturist. The envelopes were only be opened by the acupuncturist after the subject had completed baseline clinical assessments and the envelopes will be stored in a locker with no access to other personnel involved in the trial.

\section{Blinding}

Patients, investigators, outcome assessors and people responsible for the statistical analysis were blinded to the randomisation status. The acupuncturists were instructed not to exchange or reveal assignment information to other investigators or participants after he/she got randomisation status. Participants were arranged in different time schedule to avoid met with each other and information exchange. The assignment information will not be revealed until the end of the study, unless there is a serious adverse event (SAE).

\section{Data collection and management}

Study-related information such as the participant's identity, the data collected relating to the study and medical records will remain confidential. Case report forms (CRFs) will be completed on paper forms. Data will then be entered and stored in a password protected electronic database. Original paper copies of CRFs and all study data will be stored in a locker with access to the involved researchers only.

\section{Data analyses}

Based on intention-to-treat principle, all participants randomised were involved in the statistical analysis. Continuous variables will be described as means with SD. We will compare the mean changes of variables from baseline to end of intervention using analysis of covariance models that include baseline measurement as covariates. We will compare the difference of variables from baseline to the end of intervention by paired T-test or Wilcoxon rank test. Categorical variables will be presented as frequency and we will examine their differences by $\chi^{2}$ test or Fisher's exact test. $\mathrm{P}<0.05$ will be considered statistically significant. All analysis will be performed by SAS V.9.4.

\section{Safety}

All adverse events reported during the study period will be documented in CRFs.

SAE will be informed to the principal investigator within 24 hours. All SAEs must be reported on the adverse event page of the CRF. The principal investigator is responsible for the management of safety reporting according to local guidelines.

\section{ETHICS AND DISSEMINATION}

The present study is registered with ClinicalTrials.gov and conducted in accordance with the Declaration of Helsinki. The result of the present study will be published in the peer-reviewed journal.

\section{Trial status}

The recruitment phase began in July 2018. Twenty-eight patients have been recruited till now. The estimated end date for this study is in September 2019. Protocol V.1.0, $08 / 07 / 2015$.

Acknowledgements The authors thank Mrs Nan Zhao and Mrs Xue Yang for their comments and suggestions on the protocol.

Contributors $Y L$ and SL conceived the research plan. YL and $L X$ drafted the protocol. MZ, ZP, YYL and YD coordinated the study. YL, LH and MS recruited the subjects. LX, YW and $Y Q$ formed the analysis plan. All authors participated in, read and approved the final manuscript.

Funding This work was supported in part by the State Administration of Traditional Chinese Medicine of People's Republic of China (grant number JDZX2015058), National Foundation of Natural Science of China (grant number 81804139), China Scholarships Council (grant number 201808230346 and 201808230362) and Heilongjiang University of Chinese Medicine (Doctor Innovation Fund, grant number 2015bs10).

Competing interests None declared.

Patient consent for publication Not required.

Ethics approval The trial has been approved by the ethics committee of the First Affiliated Hospital of Heilongjiang University of Chinese Medicine (HZYLLKY201800301).

Provenance and peer review Not commissioned; externally peer reviewed.

Open access This is an open access article distributed in accordance with the Creative Commons Attribution Non Commercial (CC BY-NC 4.0) license, which permits others to distribute, remix, adapt, build upon this work non-commercially, and license their derivative works on different terms, provided the original work is properly cited, appropriate credit is given, any changes made indicated, and the use is non-commercial. See: http://creativecommons.org/licenses/by-nc/4.0/.

\section{REFERENCES}

1. Azziz R, Carmina E, Chen Z, et al. Polycystic ovary syndrome. Nat Rev Dis Primers 2016;2:16057.

2. Diamanti-Kandarakis E, Papavassiliou AG. Molecular mechanisms of insulin resistance in polycystic ovary syndrome. Trends $\mathrm{Mol}$ Med 2006;12:324-32.

3. Abu Hashim H. Twenty years of ovulation induction with metformin for PCOS; what is the best available evidence? Reprod Biomed Online 2016;32:44-53.

4. Graham DJ, Green L, Senior JR, et al. Troglitazone-induced liver failure: a case study. Am J Med 2003;114:299-306.

5. Yki-Järvinen $\mathrm{H}$. Thiazolidinediones. $N$ Engl J Med Overseas Ed 2004;351:1106-18.

6. Baillargeon JP, Jakubowicz DJ, luorno MJ, et al. Effects of metformin and rosiglitazone, alone and in combination, in nonobese women with polycystic ovary syndrome and normal indices of insulin sensitivity. Fertil Steril 2004;82:893-902.

7. Ortega-González C, Luna S, Hernández L, et al. Responses of serum androgen and insulin resistance to metformin and pioglitazone in obese, insulin-resistant women with polycystic ovary syndrome. $J$ Clin Endocrinol Metab 2005;90:1360-5.

8. Zheng $\mathrm{YH}$, Wang $\mathrm{XH}$, Lai MH, et al. Effectiveness of abdominal acupuncture for patients with obesity-type polycystic ovary syndrome: a randomized controlled trial. J Altern Complement Med 2013;19:740-5. 
9. Chen R, Wang C, Yan QY. Observation on therapeutic effect of polycystic ovary syndrome of damp-phlegm constitution treated with embedding therapy on backshu points and front-mu points combined with needlepricking therapy on Sifeng (EX-UE 10). Zhongguo Zhen Jiu;201:355-8.

10. Yang J, Liu Y, Huang J, et al. [Acupuncture and Chinese medicine of artificial cycle therapy for insulin resistance of polycystic ovary syndrome with phlegm damp type and its mechanism]. Zhongguo Zhen Jiu 2017;37:1163-8.

11. Song $A Q$, Zhang YP, Chen R, et al. Is Acupuncture Effective for Improving Insulin Resistance? A Systematic Review and Metaanalysis. Curr Med Sci 2018;38:1109-16.

12. Li J, Ng EH, Stener-Victorin E, et al. Acupuncture treatment for insulin sensitivity of women with polycystic ovary syndrome and insulin resistance: a study protocol for a randomized controlled trial. Trials 2017; $18: 115$.

13. Stener-Victorin $\mathrm{E}$, Zhang $\mathrm{H}$, Li R, et al. Acupuncture or metformin to improve insulin resistance in women with polycystic ovary syndrome: study protocol of a combined multinational cross sectional case-control study and a randomised controlled trial. BMJ Open 2019;9:e024733.

14. Liu CF, Yu LF, Lin CH, et al. Effect of auricular pellet acupressure on antioxidative systems in high-risk diabetes mellitus. J Altern Complement Med 2008;14:303-7.

15. Shiraishi T, Onoe M, Kageyama T, et al. Effects of auricular acupuncture stimulation on nonobese, healthy volunteer subjects. Obes Res 1995;3:667S-73.

16. Hsu CH, Wang CJ, Hwang KC, et al. The effect of auricular acupuncture in obese women: a randomized controlled trial. $J$ Womens Health 2009;18:813-8.

17. Namavar Jahromi B, Dabaghmanesh MH, Parsanezhad ME, et al. Association of leptin and insulin resistance in PCOS: A casecontrolled study. Int J Reprod Biomed 2017;15:423-8.

18. Balikci A, Erdem M, Keskin U, et al. Depression, Anxiety, and Anger in Patients with Polycystic Ovary Syndrome. Noro Psikiyatr Ars 2014;51:328-3.

19. Veltman-Verhulst SM, Boivin J, Eijkemans MJ, et al. Emotional distress is a common risk in women with polycystic ovary syndrome: a systematic review and meta-analysis of 28 studies. Hum Reprod Update 2012;18:638-51.

20. Drosdzol A, Skrzypulec V, Mazur B, et al. Quality of life and marital sexual satisfaction in women with polycystic ovary syndrome. Folia Histochem Cytobiol 2007;45(Suppl 1):S93-7.

21. Lee I, Cooney LG, Saini S, et al. Increased risk of disordered eating in polycystic ovary syndrome. Fertil Steril 2017;107:796-802.

22. Li Y, Li Y, Yu Ng EH, et al. Polycystic ovary syndrome is associated with negatively variable impacts on domains of health-related quality of life: evidence from a meta-analysis. Fertil Steril 2011;96:452-8.

23. Panico A, Messina G, Lupoli GA, et al. Quality of life in overweight (obese) and normal-weight women with polycystic ovary syndrome. Patient Prefer Adherence 2017;11:423-9.

24. Bergmann NB, Ballegaard SB, Bech PB, et al. The effect of daily self-measurement of Pressure Pain Sensitivity followed by acupressure on depression and Quality of Life (QOL), in Ischemic Heart Disease (IHD): a prospective RCT. Eur Heart $J$ 2013;34:P4311.

25. Li MK, Lee TF, Suen KP. Complementary effects of auricular acupressure in relieving constipation symptoms and promoting disease-specific health-related quality of life: A randomized placebocontrolled trial. Complement Ther Med 2014;22:266-77.

26. Zick S, Harris R. The effect of self-administered acupressure on chronic pain in breast cancer survivors. The Journal of Alternative and Complementary Medicine 2014;20:A23.

27. Wang S, Chen Z, Fu P, et al. Use of auricular acupressure to improve the quality of life in diabetic patients with chronic kidney diseases: a prospective randomized controlled trial. Evid Based Complement Alternat Med 2014;2014:1-11.

28. World Health Organization, International Association for the Study of Obesity,. International Obesity Task Force. The Asia-Pacific Perspective: Redefining Obesity and Its Treatment. Melbourne: Health Communications, Australia Pty Ltd, 2000.

29. Chen X, Yang D, Li L, et al. Abnormal glucose tolerance in Chinese women with polycystic ovary syndrome. Hum Reprod 2006;21:2027-32.

30. Zhao X, Ni R, Li L, et al. Defining hirsutism in Chinese women: a cross-sectional study. Fertil Steril 2011;96:792-6.

31. Wang FC, Tm M. Method of acupuncture and moxibustion. 4th edn. China: China Press of Traditional Chinese Medicine, 2016.

32. Ware JE, Sherbourne CD. The MOS 36-item short-form health survey (SF-36). I. Conceptual framework and item selection. Med Care 1992;30:473-83.

33. Leung KF, Liu FB, Zhao L, et al. Development and validation of the Chinese Quality of Life Instrument. Health Qual Life Outcomes 2005;3:26.

34. Nasiri-Amiri F, Ramezani Tehrani F, Simbar M, et al. Healthrelated quality of life questionnaire for polycystic ovary syndrome (PCOSQ-50): development and psychometric properties. Qual Life Res 2016;25:1791-801.

35. Whitehead AL, Julious SA, Cooper CL, et al. Estimating the sample size for a pilot randomised trial to minimise the overall trial sample size for the external pilot and main trial for a continuous outcome variable. Stat Methods Med Res 2016;25:1057-73. 